\title{
A Long-Term Comparison of Yellowstone Cutthroat Trout Abundance and Size Structure in Their Historical Range in Idaho
}

\author{
Kevin A. Meyer,* Daniel J. Schill, and F. Steven Elle \\ Idaho Department of Fish and Game, \\ 1414 East Locust Lane, \\ Nampa, Idaho 83686, USA \\ William C. SCHRADER \\ Idaho Department of Fish and Game, \\ 1515 Lincoln Road, \\ Idaho Falls, Idaho 83401, USA
}

\begin{abstract}
We compared estimates of population abundance and size structure for Yellowstone cutthroat trout Oncorhynchus clarki bouvieri obtained by electrofishing 77 stream segments across southeastern Idaho in the 1980s and again in 1999-2000 to test whether populations of Yellowstone cutthroat trout had changed. Sites sampled in the 1980s were relocated in 1999-2000 by using maps and photographs or by finding original site-boundary stakes, so that the same reach of stream was sampled during both periods. Abundance of Yellowstone cutthroat trout longer than $10 \mathrm{~cm}$ did not change, averaging $41 \mathrm{fish} / 100 \mathrm{~m}$ of stream during both the 1980s and 1999-2000. The proportion of the total catch of trout composed of Yellowstone cutthroat trout also did not change, averaging $82 \%$ in the 1980 s and $78 \%$ in $1999-2000$. At the 48 sites where size structure could be estimated for both periods, the proportion of Yellowstone cutthroat trout that were 10-20 $\mathrm{cm}$ long declined slightly ( $74 \%$ versus $66 \%$ ), but the change was due entirely to the shift in size structure at the Teton River sites. The number of sites that contained rainbow trout $O$. mykiss or cutthroat trout $\times$ rainbow trout hybrids rose from 23 to 37 , but the average proportion of the catch composed of rainbow trout and hybrids did not increase (7\% in both the 1980s and 1999-2000). Although the distribution and abundance of Yellowstone cutthroat trout have been substantially reduced in Idaho over the last century, our results indicate that Yellowstone cutthroat trout abundance and size structure in Idaho have remained relatively stable at a large number of locations for the last 10-20 years. The expanding distribution of rainbow trout and hybrids in portions of the upper Snake River basin, however, calls for additional monitoring and active management actions.
\end{abstract}

Yellowstone cutthroat trout Oncorhynchus clarki bouvieri are more abundant and have a broader distribution than any other nonanadromous cutthroat trout subspecies (Varley and Gresswell 1988; Behnke 1992). Since European settlement of the western United States, the abundance and distribution of Yellowstone cutthroat trout have declined considerably in portions of their historical range (Gresswell 1995; May 1996; Kruse et al. 2000). Factors contributing to this decline include hybridization with or displacement by nonnative trout, past overharvest from sport fishing, and habitat alterations attributable to water storage and diversion, grazing, mineral extraction, and timber harvest (Thurow et al. 1988; Varley and Gresswell 1988; Gresswell 1995). Such declines led to a pe-

\footnotetext{
* Corresponding author: kmeyer@idfg.state.id.us
}

Received July 30, 2001; accepted May 23, 2002 tition in August 1998 to list Yellowstone cutthroat trout under the Endangered Species Act (USFWS 2001).

The extent of this decline, however, remains unclear because most assessments of Yellowstone cutthroat trout status have been largely qualitative (Thurow et al. 1988; Varley and Gresswell 1988; May 1996; Thurow et al. 1997). May (1996), summarizing results from questionnaires completed by biologists with personal knowledge of localized systems, suggested that viable populations were present in only $43 \%$ of their historical range in Idaho. Thurow et al. (1997), using a similar method, estimated that Yellowstone cutthroat trout populations were strong in $32 \%$ of their entire potential range, nearly all of which occurred in Wyoming. Quantitative assessments have also focused on the proportion of historical range now occupied. For example, Kruse et al. (2000) found that $26 \%$ of the 104 trout-bearing streams in the Greybull 
and Shoshone river drainages in Wyoming outside of Yellowstone National Park contained genetically pure Yellowstone cutthroat trout.

An alternative method of assessing declines in abundance and distribution is long-term monitoring of specific populations over a broad geographic area. In 1999 and 2000, Idaho Department of Fish and Game (IDFG) personnel revisited numerous locations throughout the historical range of Yellowstone cutthroat trout in southeastern Idaho that had been sampled between 1980 and 1989. The objective of this study was to assess changes in Yellowstone cutthroat trout populations between the 1980s and 1999-2000 by comparing estimates of abundance, distribution, and size structure from these locations.

\section{Study Area}

The historical distribution of Yellowstone cutthroat trout in Idaho includes the Snake River drainage upstream from Shoshone Falls and a now extinct population from Waha Lake (Behnke 1992). The climate of the upper Snake River basin is semiarid, and many watersheds in the basin exceed 3,000 $\mathrm{m}$ in elevation. Discharge in most tributary streams is driven by snowmelt and peaks between April and June, but flows in the Snake River and South Fork Snake River are controlled by reservoir releases of irrigation water and often peak during the summer. Most streams are relatively productive for the Rocky Mountains, with conductivity exceeding $200 \mu \mathrm{S} / \mathrm{cm}$. Mountain whitefish Prosopium williamsoni is the only other salmonid native to the study area, but rainbow trout $O$. mykiss, brook trout Salvelinus fontinalis, and brown trout Salmo trutta have been introduced throughout much of the upper Snake River basin. Two species of Cottidae, three species of Catostomidae, and four species of Cyprinidae are also indigenous to the upper Snake River basin (Simpson and Wallace 1982).

The study area included a large number of individual sampling sites from six main drainages within the upper Snake River basin that were originally sampled during the 1980s with electrofishing gear to obtain Yellowstone cutthroat trout abundance estimates (Figure 1). Sites ranged from 49 to $7,300 \mathrm{~m}$ long, from 2 to $79 \mathrm{~m}$ wide, and from 1,457 to $2,097 \mathrm{~m}$ in elevation in first- to sixthorder streams.

\section{Methods}

In 1999 and 2000, IDFG personnel involved in the original sampling from 1980 to 1989 returned to identify sites and locate site boundaries. Only those sites for which survey boundaries could clearly be determined from surveyor's stakes, field notes, maps, and photographs were chosen for resampling; subsequently, 77 sites were selected for paired sampling comparisons (Figure 1). To minimize the effect that seasonal changes can have on fish abundance (Decker and Erman 1992), sampling was replicated as close to the original calendar date as possible. Sixty-five percent of the sites were resampled within 2 weeks of the original calendar date, $88 \%$ within 4 weeks, and all within 6 weeks. All sampling occurred between mid-July and early November under base flow conditions, most of the sites (71\%) being sampled in September and October.

In shallow streams less than about $8 \mathrm{~m}$ wide, two- or three-pass electrofishing removals were made by using backpack-mounted units and pulsed DC. Maximum-likelihood estimates of trout abundance and associated 95\% confidence intervals were calculated by using the MicroFish software package (Van Deventer and Platts 1989). Where all trout were captured on the first pass, confidence intervals were not estimated. For larger streams, mark-recapture electrofishing passes were made with a canoe- or boat-mounted unit and DC or pulsed DC. Log-likelihood estimates of trout abundance and associated 95\% confidence intervals were made by using the Mark Recapture for Windows software package (Montana Fish, Wildlife and Parks 1997). Mark-recapture estimates were made for each $10-\mathrm{cm}$ size-class and summed for an estimate of the total number of trout present. Total length $(\mathrm{mm})$ and weight $(\mathrm{g})$ was measured for all captured trout. Because quantitative data were not consistently collected for mountain whitefish or for nongame fish species in the 1980s, we did not include them in the analysis.

At each site, methods used to collect fish and estimate abundance in 1999-2000 mimicked those used in the 1980s with the following exceptions: at the lower and upper sites on the Blackfoot River, depletion estimates were made in 1986 and markrecapture estimates were made in 2000; at the upper site on Willow Creek, a mark-recapture estimate was made in 1984 but a depletion estimate was made in 2000; and block nets were not used at depletion-removal sites in the 1980s but were used at $39(65 \%)$ of the depletion-removal sites in 1999-2000.

Abundance estimates were made only for trout longer than $10 \mathrm{~cm}$ and were converted to numbers of fish per $100 \mathrm{~m}$ of stream. Abundance of each 


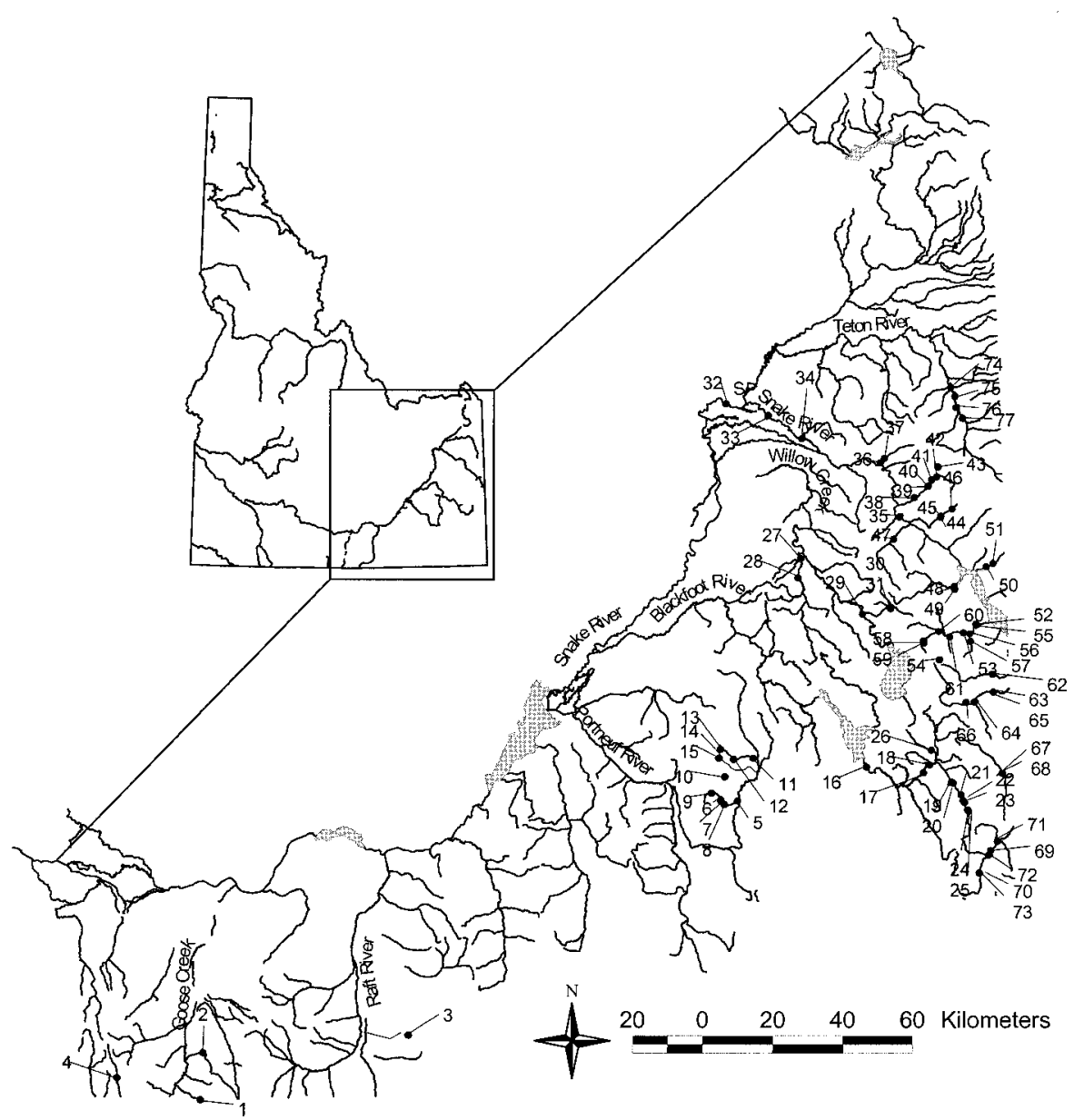

FIGURE 1.-Locations of study sites sampled in the 1980s and again in 1999-2000 across the historical range of Yellowstone cutthroat trout in Idaho. Numbers correspond to those in Table 1.

individual species of trout was estimated by multiplying the total trout abundance estimate by the proportion of the catch composed of each species. At each site, we calculated the proportion of Yellowstone cutthroat trout that were 10-20, 20-30, $30-40$, and $>40 \mathrm{~cm}$ total length and compared the proportions between periods to test for changes in Yellowstone cutthroat trout size structure. In the size-structure analysis we included only those sites where more than 20 Yellowstone cutthroat trout were caught during both sampling periods.

Many streams included in the study contained more than one sampling site. We assumed that multiple sample sites within one stream were independent samples because fish abundance did not consistently increase or decrease at sites within the same stream; of the 19 streams with more than one sampling site, nearly half (9) contained some sites that increased and others that decreased. Thus, to avoid masking the existence of such fluctuations, we did not pool sites within streams.

Yellowstone cutthroat trout, rainbow trout, and cutthroat trout $\times$ rainbow trout hybrids (hereafter called hybrids) were identified by visual examination of morphological characteristics. Yellowstone cutthroat trout were considered pure when the fish had red throat slashes, lacked white margins on the pelvic fins, and contained fewer, larger spots concentrated posteriorly. Any fish in the genus Oncorhynchus that had white fin margins, numerous spots toward the anterior of the body (especially the head area), and no or a faint red slash on the throat were pooled into a category of "rainbow trout and hybrids"; estimates of abundance and proportion of catch were made for rainbow trout and hybrids combined. 
TABLE 1.-Comparison of trout abundance estimates (fish/100 m for fish $>10 \mathrm{~cm}$ ) derived from removal (D) or mark-recapture (MR) methods at 77 study sites across southeastern Idaho between the 1980s and 1999-2000. Stream numbers correspond to Figure 1. Ninety-five percent confidence intervals (CIs) are blank where estimates were not possible; NA means not available.

\begin{tabular}{|c|c|c|c|c|c|c|c|c|c|c|}
\hline \multirow[b]{3}{*}{ Site } & \multirow[b]{3}{*}{ Stream ${ }^{\mathrm{a}}$} & \multirow{2}{*}{\multicolumn{2}{|c|}{ Study site }} & & \multicolumn{6}{|c|}{ Total trout abundance } \\
\hline & & & & & \multicolumn{3}{|c|}{$1980 \mathrm{~s}$} & \\
\hline & & $\begin{array}{l}\text { Length } \\
\text { (m) }\end{array}$ & $\begin{array}{l}\text { Width } \\
\text { (m) }\end{array}$ & Method & Estimate & $95 \% \mathrm{CI}$ & Year & Estimate & $95 \% \mathrm{CI}$ & Year \\
\hline \multicolumn{11}{|c|}{ Raft River and Goose Creek drainages } \\
\hline 1 & Birch Creek & 80 & 1.9 & $\mathrm{D}$ & 3 & & 1987 & 31 & $31-31$ & 2000 \\
\hline 2 & Cold Creek & 60 & 1.5 & $\mathrm{D}$ & 5 & $5-10$ & 1987 & 6 & $6-9$ & 2000 \\
\hline 3 & Eightmile Creek & 82 & 1.3 & $\mathrm{D}$ & 6 & $6-8$ & 1986 & 23 & $23-25$ & 2000 \\
\hline 4 & Trout Creek & 110 & 3.2 & $\mathrm{D}$ & 55 & & 1987 & 8 & $8-9$ & 2000 \\
\hline \multicolumn{11}{|c|}{ Portneuf River drainage } \\
\hline 5 & Pebble Creek & 207 & 2.3 & $\mathrm{D}$ & 59 & $57-62$ & 1986 & 15 & & 2000 \\
\hline 6 & Pebble Creek & 98 & 4.6 & $\mathrm{D}$ & 39 & $38-43$ & 1986 & 52 & $52-54$ & 1999 \\
\hline 7 & Pebble Creek & 104 & 3.6 & $\mathrm{D}$ & 80 & $77-85$ & 1986 & 58 & $58-59$ & 1999 \\
\hline 8 & Pebble Creek, NF & 133 & 1.8 & $\mathrm{D}$ & 35 & $35-36$ & 1986 & 15 & & 1999 \\
\hline 9 & Big Springs Creek & 105 & NA & $\mathrm{D}$ & 29 & $29-30$ & 1986 & 28 & $28-30$ & 1999 \\
\hline 10 & King Creek & 70 & 11.3 & $\mathrm{D}$ & 7 & $7-9$ & 1986 & 0 & & 2000 \\
\hline 11 & Toponce Creek & 180 & 1.7 & $\mathrm{D}$ & 1 & & 1986 & 0 & & 2000 \\
\hline 12 & Toponce Creek & 89 & 6.6 & $\mathrm{D}$ & 112 & $101-127$ & 1986 & 35 & $35-37$ & 2000 \\
\hline 13 & Toponce Creek, MF & 80 & NA & $\mathrm{D}$ & 79 & $79-81$ & 1986 & 96 & $96-97$ & 2000 \\
\hline 14 & Toponce Creek, SF & 99 & 2.7 & $\mathrm{D}$ & 134 & & 1987 & 47 & $45-53$ & 2000 \\
\hline 15 & Toponce Creek, SF & 113 & 7.2 & $\mathrm{D}$ & 31 & $31-32$ & 1987 & 154 & $150-159$ & 2000 \\
\hline \multicolumn{11}{|c|}{ Blackfoot River drainage } \\
\hline 16 & Blackfoot River & 4,720 & NA & $\mathrm{D} / \mathrm{MR}$ & 1 & $1-1$ & 1988 & 14 & $12-17$ & 2000 \\
\hline 17 & Blackfoot River & 1,712 & NA & MR & 15 & $12-19$ & 1988 & 44 & $39-48$ & 2000 \\
\hline 18 & Blackfoot River & 1,760 & NA & $\mathrm{D} / \mathrm{MR}$ & 6 & $6-7$ & 1988 & 20 & $13-27$ & 2000 \\
\hline 19 & Diamond Creek & 180 & 4.8 & $\mathrm{D}$ & 10 & $10-11$ & 1988 & 11 & $11-12$ & 2000 \\
\hline 20 & Diamond Creek & 147 & 5.5 & $\mathrm{D}$ & 135 & $131-140$ & 1980 & 69 & $69-70$ & 2000 \\
\hline 21 & Diamond Creek & 150 & 4.1 & $\mathrm{D}$ & 176 & $172-180$ & 1980 & 67 & $67-69$ & 2000 \\
\hline 22 & Diamond Creek & 165 & 3.4 & $\mathrm{D}$ & 27 & $25-33$ & 1987 & 71 & $70-73$ & 2000 \\
\hline 23 & Diamond Creek & 87 & 3.3 & $\mathrm{D}$ & 12 & $12-15$ & 1987 & 48 & $48-49$ & 2000 \\
\hline 24 & Diamond Creek & 75 & 2.4 & $\mathrm{D}$ & 52 & $52-54$ & 1987 & 56 & $47-77$ & 2000 \\
\hline 25 & Diamond Creek & 165 & 2.8 & $\mathrm{D}$ & 23 & $23-23$ & 1988 & 48 & $44-54$ & 2000 \\
\hline 26 & Sheep Creek & 161 & 2.7 & $\mathrm{D}$ & 5 & & 1987 & 6 & $6-7$ & 2000 \\
\hline \multicolumn{11}{|c|}{ Willow Creek drainage } \\
\hline 27 & Willow Creek & 886 & 8.2 & MR & 25 & $19-32$ & 1984 & 5 & $3-6$ & 2000 \\
\hline 28 & Willow Creek & 571 & NA & MR/D & 92 & $75-109$ & 1984 & 25 & $21-30$ & 2000 \\
\hline 29 & Brockman Creek & 93 & NA & $\mathrm{D}$ & 10 & $10-10$ & 1983 & 27 & $25-34$ & 2000 \\
\hline 30 & Corral Creek & 71 & 1.2 & $\mathrm{D}$ & 65 & $52-89$ & 1982 & 6 & $6-8$ & 2000 \\
\hline 31 & Corral Creek & 127 & 2.0 & $\mathrm{D}$ & 28 & $28-29$ & 1982 & 15 & $15-16$ & 2000 \\
\hline \multicolumn{11}{|c|}{ Main-stem Snake River drainage } \\
\hline 32 & Snake River & 7,300 & 79.0 & MR & 21 & $6-55$ & 1988 & 41 & $8-135$ & 2000 \\
\hline \multicolumn{11}{|c|}{ South Fork Snake River drainage } \\
\hline 33 & Snake River, SF & 4,800 & 46.0 & MR & 64 & $11-186$ & 1989 & 147 & $30-300$ & 1999 \\
\hline 34 & Snake River, SF & 2,900 & 66.0 & MR & 95 & $25-200$ & 1989 & 260 & $58-463$ & 2000 \\
\hline 35 & Snake River, SF & 4,900 & 71.0 & MR & 180 & $52-303$ & 1989 & 282 & $58-514$ & 1999 \\
\hline 36 & Burns Creek & 85 & 5.9 & $\mathrm{D}$ & 57 & $53-65$ & 1980 & 54 & $45-71$ & 2000 \\
\hline 37 & Burns Creek & 86 & 5.3 & $\mathrm{D}$ & 7 & $7-8$ & 1980 & 37 & $37-39$ & 2000 \\
\hline 38 & Pine Creek & 66 & 11.0 & $\mathrm{D}$ & 52 & $50-57$ & 1980 & 77 & $74-85$ & 2000 \\
\hline 39 & Pine Creek & 90 & 10.6 & $\mathrm{D}$ & 78 & $78-79$ & 1988 & 24 & $24-27$ & 2000 \\
\hline 40 & Pine Creek & 74 & 5.1 & $\mathrm{D}$ & 155 & $135-182$ & 1980 & 152 & $129-178$ & 2000 \\
\hline 41 & Pine Creek & 80 & 4.9 & $\mathrm{D}$ & 54 & $54-56$ & 1988 & 90 & $90-92$ & 2000 \\
\hline 42 & Pine Creek, NF & 72 & 5.4 & $\mathrm{D}$ & 22 & $22-24$ & 1982 & 18 & $18-19$ & 2000 \\
\hline 43 & Pine Creek, NF & 80 & 7.9 & $\mathrm{D}$ & 44 & $44-47$ & 1981 & 10 & $10-13$ & 2000 \\
\hline 44 & Rainey Creek & 160 & 5.7 & $\mathrm{D}$ & 1 & & 1980 & 39 & $34-48$ & 2000 \\
\hline 45 & Rainey Creek & 123 & 6.1 & $\mathrm{D}$ & 7 & $7-9$ & 1980 & 4 & $4-7$ & 2000 \\
\hline 46 & Rainey Creek & 167 & 7.8 & $\mathrm{D}$ & 13 & $13-14$ & 1980 & 53 & $53-54$ & 2000 \\
\hline 47 & Fall Creek & 133 & 6.0 & $\mathrm{D}$ & 13 & $13-15$ & 1988 & 61 & $54-71$ & 2000 \\
\hline 48 & Bear Creek & 246 & 8.3 & $\mathrm{D}$ & 18 & $10-42$ & 1980 & 72 & $70-74$ & 2000 \\
\hline 49 & Elk Creek & 146 & 3.6 & $\mathrm{D}$ & 25 & $19-38$ & 1980 & 36 & $36-39$ & 2000 \\
\hline
\end{tabular}


TABLE 1.-Extended.

\begin{tabular}{|c|c|c|c|c|c|c|c|c|c|}
\hline \multirow[b]{3}{*}{ Site } & \multirow[b]{3}{*}{ Stream $^{\mathrm{a}}$} & \multicolumn{8}{|c|}{ Individual species abundance } \\
\hline & & \multicolumn{2}{|c|}{$\begin{array}{l}\text { Yellowstone } \\
\text { cutthroat trout }\end{array}$} & \multicolumn{2}{|c|}{$\begin{array}{l}\text { Rainbow trout } \\
\text { and hybrids }\end{array}$} & \multicolumn{2}{|c|}{ Brown trout } & \multicolumn{2}{|c|}{ Brook trout } \\
\hline & & $1980 \mathrm{~s}$ & $\begin{array}{l}1999- \\
2000\end{array}$ & $1980 \mathrm{~s}$ & $\begin{array}{l}1999- \\
2000\end{array}$ & $1980 \mathrm{~s}$ & $\begin{array}{l}1999- \\
2000\end{array}$ & $1980 \mathrm{~s}$ & $\begin{array}{c}1999- \\
2000\end{array}$ \\
\hline \multicolumn{10}{|c|}{ Raft River and Goose Creek drainages } \\
\hline 1 & Birch Creek & 1 & 0 & & & & & 1 & 31 \\
\hline 2 & Cold Creek & 5 & 0 & & & & & 0 & 6 \\
\hline 3 & Eightmile Creek & 6 & 23 & & & & & & \\
\hline 4 & Trout Creek & 4 & 7 & 51 & 1 & & & & \\
\hline \multicolumn{10}{|c|}{ Portneuf River drainage } \\
\hline 5 & Pebble Creek & 44 & 14 & 14 & 1 & & & 1 & 0 \\
\hline 6 & Pebble Creek & 33 & 45 & 6 & 7 & & & & \\
\hline 7 & Pebble Creek & 76 & 44 & 4 & 14 & & & & \\
\hline 8 & Pebble Creek, NF & 35 & 15 & & & & & & \\
\hline 9 & Big Springs Creek & 24 & 26 & 5 & 2 & & & & \\
\hline 10 & King Creek & 7 & 0 & & & & & & \\
\hline 11 & Toponce Creek & 1 & 0 & & & & & & \\
\hline 12 & Toponce Creek & 8 & 5 & 105 & 1 & 0 & 29 & & \\
\hline 13 & Toponce Creek, MF & 3 & 17 & 76 & 78 & & & & \\
\hline 14 & Toponce Creek, SF & 116 & 46 & 19 & 1 & & & & \\
\hline 15 & Toponce Creek, SF & 29 & 147 & 2 & 7 & & & & \\
\hline \multicolumn{10}{|c|}{ Blackfoot River drainage } \\
\hline 16 & Blackfoot River & 1 & 13 & 0 & 2 & & & & \\
\hline 17 & Blackfoot River & 15 & 37 & 0 & 7 & & & 0 & 0 \\
\hline 18 & Blackfoot River & 6 & 13 & 0 & 6 & & & 0 & 1 \\
\hline 19 & Diamond Creek & 8 & 11 & & & & & 1 & 0 \\
\hline 20 & Diamond Creek & 130 & 62 & 0 & 7 & & & 5 & 0 \\
\hline 21 & Diamond Creek & 174 & 61 & 0 & 6 & & & 1 & 0 \\
\hline 22 & Diamond Creek & 25 & 64 & 0 & 7 & & & 3 & 0 \\
\hline 23 & Diamond Creek & 9 & 44 & 0 & 5 & & & 2 & 0 \\
\hline 24 & Diamond Creek & 51 & 44 & 0 & 12 & & & 1 & 0 \\
\hline 25 & Diamond Creek & 18 & 29 & 0 & 19 & & & 5 & 0 \\
\hline 26 & Sheep Creek & 5 & 6 & & & & & & \\
\hline \multicolumn{10}{|c|}{ Willow Creek drainage } \\
\hline 27 & Willow Creek & 21 & 4 & 1 & 0 & 3 & 1 & & \\
\hline 28 & Willow Creek & 67 & 23 & 1 & 0 & 24 & 2 & & \\
\hline 29 & Brockman Creek & 8 & 27 & & & 2 & 0 & & \\
\hline 30 & Corral Creek & 65 & 6 & & & & & & \\
\hline 31 & Corral Creek & 28 & 15 & & & & & & \\
\hline \multicolumn{10}{|c|}{ Main-stem Snake River drainage } \\
\hline 32 & Snake River & 8 & 8 & 3 & 3 & 10 & 30 & & \\
\hline \multicolumn{10}{|c|}{ South Fork Snake River drainage } \\
\hline 33 & Snake River, SF & 21 & 34 & 0 & 1 & 42 & 112 & & \\
\hline 34 & Snake River, SF & 48 & 73 & 0 & 2 & 47 & 185 & & \\
\hline 35 & Snake River, SF & 160 & 177 & 7 & 55 & 12 & 51 & & \\
\hline 36 & Burns Creek & 57 & 33 & 0 & 20 & & & & \\
\hline 37 & Burns Creek & 7 & 31 & 0 & 2 & 0 & 4 & & \\
\hline 38 & Pine Creek & 52 & 71 & 0 & 6 & & & & \\
\hline 39 & Pine Creek & 78 & 24 & & & & & & \\
\hline 40 & Pine Creek & 155 & 147 & 0 & 5 & & & & \\
\hline 41 & Pine Creek & 54 & 83 & 0 & 8 & & & & \\
\hline 42 & Pine Creek, NF & 22 & 18 & & & & & & \\
\hline 43 & Pine Creek, NF & 44 & 9 & 0 & 1 & & & & \\
\hline 44 & Rainey Creek & 1 & 38 & & & 0 & 2 & & \\
\hline 45 & Rainey Creek & 7 & 4 & & & & & & \\
\hline 46 & Rainey Creek & 9 & 40 & 0 & 2 & 4 & 11 & & \\
\hline 47 & Fall Creek & 13 & 61 & & & & & & \\
\hline 48 & Bear Creek & 18 & 72 & 0 & 0 & & & & \\
\hline 49 & Elk Creek & 25 & 36 & & & & & & \\
\hline
\end{tabular}


TABLE 1.-Continued.

\begin{tabular}{|c|c|c|c|c|c|c|c|c|c|c|}
\hline \multirow[b]{4}{*}{ Site } & \multirow[b]{4}{*}{ Stream $^{\mathrm{a}}$} & \multirow{2}{*}{\multicolumn{2}{|c|}{ Study site }} & \multirow[b]{4}{*}{ Method } & \multicolumn{6}{|c|}{ Total trout abundance } \\
\hline & & & & & \multirow{2}{*}{\multicolumn{3}{|c|}{$1980 \mathrm{~s}$}} & \multirow{2}{*}{\multicolumn{3}{|c|}{ 1999-2000 }} \\
\hline & & \multirow[b]{2}{*}{$\begin{array}{l}\text { Length } \\
\text { (m) }\end{array}$} & \multirow[b]{2}{*}{$\begin{array}{l}\text { Width } \\
\text { (m) }\end{array}$} & & & & & & & \\
\hline & & & & & Estimate & $95 \% \mathrm{CI}$ & Year & Estimate & $95 \%$ CI & Year \\
\hline 50 & Big Elk Creek & 97 & 6.9 & $\mathrm{D}$ & 8 & $8-9$ & 1980 & 33 & $32-37$ & 2000 \\
\hline 51 & Big Elk Creek & 146 & 7.7 & $\mathrm{D}$ & 20 & $17-27$ & 1980 & 61 & $58-66$ & 2000 \\
\hline 52 & McCoy Creek & 375 & 9.3 & MR & 73 & $47-100$ & 1986 & 37 & $33-40$ & 2000 \\
\hline 53 & McCoy Creek & 388 & 8.7 & MR & 109 & $85-132$ & 1986 & 118 & $79-156$ & 2000 \\
\hline 54 & McCoy Creek & 144 & 3.3 & $\mathrm{D}$ & 53 & $50-58$ & 1986 & 57 & $55-61$ & 1999 \\
\hline 55 & Jensen Creek & 49 & 3.6 & $\mathrm{D}$ & 163 & $92-304$ & 1986 & 0 & & 1999 \\
\hline 56 & Fish Creek & 79 & 1.9 & $\mathrm{D}$ & 48 & $45-57$ & 1986 & 167 & $163-173$ & 1999 \\
\hline 57 & Fish Creek & 92 & 3.2 & $\mathrm{D}$ & 44 & $42-47$ & 1986 & 90 & 86-97 & 1999 \\
\hline 58 & Barnes Creek & 99 & 2.7 & $\mathrm{D}$ & 24 & $24-26$ & 1986 & 34 & $28-45$ & 1999 \\
\hline 59 & Barnes Creek & 76 & 3.2 & $\mathrm{D}$ & 8 & $8-10$ & 1986 & 11 & & 1999 \\
\hline 60 & Clear Creek & 122 & 3.2 & $\mathrm{D}$ & 62 & $38-107$ & 1986 & 31 & $30-34$ & 1999 \\
\hline 61 & Iowa Creek & 97 & 3.6 & $\mathrm{D}$ & 26 & $26-27$ & 1986 & 31 & $31-33$ & 2000 \\
\hline 62 & Jackknife Creek & 107 & 5.7 & $\mathrm{D}$ & 30 & $30-31$ & 1987 & 14 & $14-15$ & 1999 \\
\hline 63 & Tincup Creek & 155 & 6.3 & $\mathrm{D}$ & 64 & $56-75$ & 1987 & 77 & $73-84$ & 1999 \\
\hline 64 & Tincup Creek & 117 & 6.8 & $\mathrm{D}$ & 133 & $128-139$ & 1987 & 64 & $62-68$ & 1999 \\
\hline 65 & Tincup Creek & 100 & 5.1 & $\mathrm{D}$ & 66 & $65-69$ & 1987 & 21 & $21-23$ & 1999 \\
\hline 66 & Bear Canyon Creek & 52 & 1.8 & $\mathrm{D}$ & 88 & $88-92$ & 1987 & 34 & $34-37$ & 1999 \\
\hline 67 & Stump Creek & 441 & 7.0 & MR & 54 & $43-66$ & 1986 & 149 & $135-163$ & 2000 \\
\hline 68 & Horse Creek & 86 & 2.2 & $\mathrm{D}$ & 41 & $41-43$ & 1986 & 75 & $75-77$ & 1999 \\
\hline 69 & Crow Creek & 309 & 5.3 & MR & 9 & $8-10$ & 1986 & 43 & $38-47$ & 2000 \\
\hline 70 & Crow Creek & 111 & 4.2 & $\mathrm{D}$ & 85 & $82-90$ & 1986 & 126 & $126-128$ & 1999 \\
\hline 71 & Sage Creek & 215 & 5.7 & $\mathrm{D}$ & 120 & $107-132$ & 1987 & 140 & $139-141$ & 1999 \\
\hline 72 & Deer Creek & 157 & NA & $\mathrm{D}$ & 45 & $38-56$ & 1986 & 85 & $85-86$ & 1999 \\
\hline 73 & White Dugway Creek & 84 & 1.8 & $\mathrm{D}$ & 14 & $14-17$ & 1986 & 6 & & 1999 \\
\hline \multicolumn{11}{|c|}{ Teton River drainage } \\
\hline 74 & Teton River & 4,900 & 26.0 & MR & 69 & $8-363$ & 1987 & 18 & $5-66$ & 1999 \\
\hline 75 & Teton River & 5,500 & 37.0 & MR & 59 & $10-196$ & 1987 & 25 & $4-105$ & 2000 \\
\hline 76 & Teton River & 7,100 & 37.0 & MR & 54 & $10-174$ & 1987 & 25 & $4-116$ & 2000 \\
\hline 77 & Teton River & 5,800 & 42.0 & MR & 78 & $10-338$ & 1987 & 19 & $6-45$ & 1999 \\
\hline \multicolumn{2}{|c|}{ Average } & & & & 51 & & & 54 & & \\
\hline
\end{tabular}

${ }^{\mathrm{a}} \mathrm{NF}=$ North Fork, $\mathrm{MF}=$ Middle Fork, and $\mathrm{SF}=$ South Fork.

Fin clips were collected randomly from individuals of Oncorhynchus spp. at all locations. Genetic analysis for 17 arbitrarily selected sites was performed by the Aquaculture Research Institute at the University of Idaho, which followed procedures described by Campbell et al. (2002). The extent of rainbow trout introgression occurring at the study sites was assessed genetically by using species-specific restriction fragment length polymorphisms (RFLPs) of nuclear DNA (nDNA) and mitochondrial DNA (mtDNA) gene loci. Fish identified with rainbow trout nDNA or mtDNA were assigned to the category "rainbow trout and hybrids." Results, reported as the proportion of individual Oncorhynchus fin clips that contained rainbow trout nDNA or mtDNA, were compared against our visual estimate of the rate of rainbow trout introgression (i.e., the proportion of the total catch of Oncorhynchus composed of rainbow trout and hybrids) based on phenotypic characteristics.

We tested whether Yellowstone cutthroat trout abundance or proportional size structure had changed from the 1980s to $1999-2000$ by using paired $t$-tests (Zar 1996) and 95\% confidence intervals around $\bar{d}$, the difference between means (Johnson 1995). Proportional data are known to be binomially rather than normally distributed. However, data do not need to be normally distributed for the $t$-test to apply; only the means need to be, and that property is assured by the Central Limit Theorem (Johnson 1995). Therefore, we made no transformation to the percentage data. However, because the four stock structure comparisons were not independent, we used the Bonferroni method (Sokal and Rohlf 1987) of reducing the significance value for each comparison so that the experimental type I error rate $(\alpha)$ did not exceed 0.05 ; thus the adjusted $\alpha=0.05 \div 4=$ 0.0125 .

\section{Results}

Yellowstone cutthroat trout abundance at the 77 paired sites was not different between time peri- 
TABLE 1.-Extended. (Continued)

\begin{tabular}{|c|c|c|c|c|c|c|c|c|c|}
\hline \multirow[b]{3}{*}{ Site } & \multirow[b]{3}{*}{ Stream $^{\mathrm{a}}$} & \multicolumn{8}{|c|}{ Individual species abundance } \\
\hline & & \multicolumn{2}{|c|}{$\begin{array}{l}\text { Yellowstone } \\
\text { cutthroat trout }\end{array}$} & \multicolumn{2}{|c|}{$\begin{array}{l}\text { Rainbow trout } \\
\text { and hybrids }\end{array}$} & \multicolumn{2}{|c|}{ Brown trout } & \multicolumn{2}{|c|}{ Brook trout } \\
\hline & & $1980 \mathrm{~s}$ & $\begin{array}{c}1999- \\
2000\end{array}$ & $1980 \mathrm{~s}$ & $\begin{array}{c}1999- \\
2000\end{array}$ & $1980 \mathrm{~s}$ & $\begin{array}{c}1999- \\
2000\end{array}$ & $1980 \mathrm{~s}$ & $\begin{array}{c}1999- \\
2000\end{array}$ \\
\hline 50 & Big Elk Creek & 8 & 33 & & & & & & \\
\hline 51 & Big Elk Creek & 20 & 61 & & & & & & \\
\hline 52 & McCoy Creek & 72 & 36 & 0 & 0 & 1 & 1 & & \\
\hline 53 & McCoy Creek & 108 & 118 & & & 1 & 0 & & \\
\hline 54 & McCoy Creek & 53 & 57 & & & & & & \\
\hline 55 & Jensen Creek & 163 & 0 & & & & & & \\
\hline 56 & Fish Creek & 48 & 167 & & & & & & \\
\hline 57 & Fish Creek & 44 & 90 & & & & & & \\
\hline 58 & Barnes Creek & 24 & 34 & & & & & & \\
\hline 59 & Barnes Creek & 8 & 11 & & & & & & \\
\hline 60 & Clear Creek & 62 & 31 & & & & & & \\
\hline 61 & Iowa Creek & 26 & 31 & & & & & & \\
\hline 62 & Jackknife Creek & 29 & 14 & & & 1 & 0 & & \\
\hline 63 & Tincup Creek & 63 & 76 & 1 & 0 & 1 & 1 & & \\
\hline 64 & Tincup Creek & 129 & 64 & 1 & 0 & 3 & 0 & & \\
\hline 65 & Tincup Creek & 66 & 21 & & & & & & \\
\hline 66 & Bear Canyon Creek & 88 & 32 & 0 & 2 & & & & \\
\hline 67 & Stump Creek & 44 & 124 & & & 10 & 26 & 1 & 0 \\
\hline 68 & Horse Creek & 41 & 71 & & & 0 & 5 & & \\
\hline 69 & Crow Creek & 4 & 10 & & & 5 & 32 & & \\
\hline 70 & Crow Creek & 84 & 117 & & & 1 & 9 & & \\
\hline 71 & Sage Creek & 19 & 31 & & & 100 & 109 & & \\
\hline 72 & Deer Creek & 37 & 79 & 0 & 1 & 8 & 6 & & \\
\hline 73 & White Dugway Creek & 13 & 6 & & & 1 & 0 & & \\
\hline \multicolumn{10}{|c|}{ Teton River drainage } \\
\hline 74 & Teton River & 12 & 8 & 46 & 7 & & & 12 & 3 \\
\hline 75 & Teton River & 16 & 14 & 29 & 7 & & & 14 & 4 \\
\hline 76 & Teton River & 25 & 15 & 17 & 7 & 0 & 0 & 12 & 4 \\
\hline 77 & Teton River & 42 & 11 & 5 & 2 & & & 31 & 6 \\
\hline Aver & & 41 & 41 & 5 & 4 & 4 & 8 & 1 & 1 \\
\hline
\end{tabular}

ods, averaging $41 \mathrm{fish} / 100 \mathrm{~m}$ of stream in both the $1980 \mathrm{~s}$ and $1999-2000(\bar{d}=0.3 \pm 8.7 ; t=-0.05$; $P=0.96$; Table 1). Abundance was lower than in 1999-2000 at 33 locations and higher at 44 locations. We also found no differences between time periods within individual drainages (Table 2), but sample sizes were low for most of these comparisons. At five locations, no Yellowstone cutthroat trout longer than $10 \mathrm{~cm}$ were captured in
1999-2000 where they had been captured in the 1980 s, but one of these sites did contain Yellowstone cutthroat trout smaller than $10 \mathrm{~cm}$. Overall trout abundance also remained relatively unchanged between time periods, averaging 51 fish/ $100 \mathrm{~m}$ in the 1980 s, compared with 54 in 1999 2000 (Table 1).

Yellowstone cutthroat trout on average made up a similar proportion of the catch in the $1980 \mathrm{~s}$

TABLE 2.- Sample size and mean abundance (fish/100 $\mathrm{m}$ for fish $>10 \mathrm{~cm}$ ) of Yellowstone cutthroat trout in Idaho and $t$-test summary statistics by drainage; $\bar{d}$ is the difference between the mean for the 1980s and that for 1999-2000.

\begin{tabular}{|c|c|c|c|c|}
\hline \multirow[b]{2}{*}{ Drainage } & \multirow[b]{2}{*}{$n$} & \multicolumn{2}{|c|}{ Mean abundance } & \multirow[b]{2}{*}{$\bar{d} \pm 95 \% \mathrm{CI}$} \\
\hline & & $1980 \mathrm{~s}$ & 1999-2000 & \\
\hline Raft River and Goose Creek & 4 & 4 & 8 & $4 \pm 15$ \\
\hline Portneuf River & 11 & 34 & 33 & $-1 \pm 32$ \\
\hline Blackfoot River & 11 & 40 & 35 & $-5 \pm 39$ \\
\hline Willow Creek & 5 & 38 & 15 & $-23 \pm 37$ \\
\hline South Fork Snake River & 41 & 49 & 55 & $6 \pm 14$ \\
\hline Teton River & 4 & 24 & 12 & $-12 \pm 21$ \\
\hline Total & 77 & 41 & 41 & $0.3 \pm 10$ \\
\hline
\end{tabular}


TABLE 3.-Comparison of the estimates of genetic and visual rates of introgression at the study sites. The genetic rate of introgression is the proportion of individual fin clips from Oncorhynchus spp. containing rainbow trout nuclear DNA (nDNA) or mitochondrial DNA (mtDNA), and the visual rate of introgression is the proportion of the total catch of Oncorhynchus spp. with phenotypic characteristics of rainbow trout and hybrids. Yellowstone cutthroat trout populations in which no hybridization was detected have introgression rates of zero; $n$ is the sample size.

\begin{tabular}{|c|c|c|c|c|c|c|}
\hline \multirow[b]{2}{*}{ Site } & \multirow[b]{2}{*}{ Stream $^{\mathrm{a}}$} & \multicolumn{3}{|c|}{$\begin{array}{l}\text { Genetic rates of } \\
\text { introgression }\end{array}$} & \multicolumn{2}{|c|}{$\begin{array}{l}\text { Visual rates of } \\
\text { introgression }\end{array}$} \\
\hline & & $n$ & nDNA & mtDNA & $1980 \mathrm{~s}$ & 1999-2000 \\
\hline \multicolumn{7}{|c|}{ Raft River and Goose Creek drainages } \\
\hline 1 & Birch Creek & & & & 0 & \\
\hline 2 & Cold Creek & & & & 0 & 100 \\
\hline 3 & Eightmile Creek & 20 & 0 & 0 & 0 & 0 \\
\hline 4 & Trout Creek & & & & 92 & 11 \\
\hline \multicolumn{7}{|c|}{ Portneuf River drainage } \\
\hline 5 & Pebble Creek & & & & 24 & 6 \\
\hline 6 & Pebble Creek & & & & 16 & 14 \\
\hline 7 & Pebble Creek & & & & 5 & 23 \\
\hline 8 & Pebble Creek, NF & 47 & 2.1 & 0 & 0 & 0 \\
\hline 9 & Big Springs Creek & 48 & 4.3 & 0 & 13 & 7 \\
\hline 10 & King Creek & & & & 0 & \\
\hline 11 & Toponce Creek & & & & 0 & \\
\hline 12 & Toponce Creek & & & & 93 & 19 \\
\hline 13 & Toponce Creek, MF & & & & 96 & 84 \\
\hline 14 & Toponce Creek, SF & & & & 12 & 5 \\
\hline 15 & Toponce Creek, SF & & & & 6 & 2 \\
\hline \multicolumn{7}{|c|}{ Blackfoot River drainage } \\
\hline 16 & Blackfoot River & & & & 0 & 13 \\
\hline 17 & Blackfoot River & 24 & 25.0 & 20.8 & 1 & 16 \\
\hline 18 & Blackfoot River & & & & 2 & 33 \\
\hline 19 & Diamond Creek & & & & 0 & 0 \\
\hline 20 & Diamond Creek & & & & 0 & 10 \\
\hline 21 & Diamond Creek & & & & 0 & 9 \\
\hline 22 & Diamond Creek & & & & 0 & 9 \\
\hline 23 & Diamond Creek & & & & 0 & 10 \\
\hline 24 & Diamond Creek & & & & 0 & 23 \\
\hline 25 & Diamond Creek & & & & 0 & 39 \\
\hline 26 & Sheep Creek & & & & 0 & 0 \\
\hline \multicolumn{7}{|c|}{ Willow Creek drainage } \\
\hline 27 & Willow Creek & 46 & 0 & 0 & 6 & 0 \\
\hline 28 & Willow Creek & & & & 1 & 0 \\
\hline 29 & Brockman Creek & & & & 0 & 0 \\
\hline 30 & Corral Creek & & & & 0 & 0 \\
\hline 31 & Corral Creek & & & & 0 & 0 \\
\hline \multicolumn{7}{|c|}{ Main-stem Snake River drainage } \\
\hline 32 & Snake River & & & & 27 & 29 \\
\hline \multicolumn{7}{|c|}{ South Fork Snake River drainage } \\
\hline 33 & Snake River, SF & & & & 3 & 4 \\
\hline 34 & Snake River, SF & & & & 0 & 3 \\
\hline 35 & Snake River, SF & & & & 4 & 24 \\
\hline 36 & Burns Creek & 48 & 6.3 & 2.1 & 0 & 11 \\
\hline 37 & Burns Creek & & & & 0 & 7 \\
\hline 38 & Pine Creek & & & & 0 & 8 \\
\hline 39 & Pine Creek & & & & 0 & 0 \\
\hline 40 & Pine Creek & & & & 0 & 3 \\
\hline 41 & Pine Creek & 47 & 22.9 & 13.0 & 0 & 8 \\
\hline 42 & Pine Creek, NF & & & & 0 & 0 \\
\hline 43 & Pine Creek, NF & & & & 0 & 12 \\
\hline 44 & Rainey Creek & & & & 0 & 0 \\
\hline 45 & Rainey Creek & & & & 0 & 0 \\
\hline 46 & Rainey Creek & & & & 0 & 4 \\
\hline 47 & Fall Creek & & & & 0 & 0 \\
\hline 48 & Bear Creek & & & & 0 & 1 \\
\hline 49 & Elk Creek & & & & 0 & 0 \\
\hline 50 & Big Elk Creek & 30 & 0 & 0 & 0 & 0 \\
\hline
\end{tabular}


TABLE 3.-Continued.

\begin{tabular}{|c|c|c|c|c|c|c|}
\hline \multirow[b]{2}{*}{ Site } & \multirow[b]{2}{*}{ Stream $^{\mathrm{a}}$} & \multicolumn{3}{|c|}{$\begin{array}{c}\text { Genetic rates of } \\
\text { introgression }\end{array}$} & \multicolumn{2}{|c|}{$\begin{array}{l}\text { Visual rates of } \\
\text { introgression }\end{array}$} \\
\hline & & $n$ & nDNA & mtDNA & $1980 \mathrm{~s}$ & $1999-2000$ \\
\hline 51 & Big Elk Creek & & & & 0 & 0 \\
\hline 52 & McCoy Creek & & & & 0 & 1 \\
\hline 53 & McCoy Creek & & & & 0 & 0 \\
\hline 54 & McCoy Creek & 38 & 0 & 0 & 0 & 0 \\
\hline 55 & Jensen Creek & 30 & $0^{\mathrm{b}}$ & $0^{\mathrm{b}}$ & 0 & $0^{\mathrm{b}}$ \\
\hline 56 & Fish Creek & 48 & 0 & 0 & 0 & 0 \\
\hline 57 & Fish Creek & & & & 0 & 0 \\
\hline 58 & Barnes Creek & 45 & 0 & 0 & 0 & 0 \\
\hline 59 & Barnes Creek & & & & 0 & 0 \\
\hline 60 & Clear Creek & 48 & 0 & 0 & 0 & 0 \\
\hline 61 & Iowa Creek & & & & 0 & 0 \\
\hline 62 & Jackknife Creek & & & & 0 & 0 \\
\hline 63 & Tincup Creek & & & & 1 & 0 \\
\hline 64 & Tincup Creek & & & & 1 & 0 \\
\hline 65 & Tincup Creek & 48 & 0 & 0 & 0 & 0 \\
\hline 66 & Bear Canyon Creek & & & & 0 & 6 \\
\hline 67 & Stump Creek & & & & 0 & 0 \\
\hline 68 & Horse Creek & 48 & 0 & 0 & 0 & 0 \\
\hline 69 & Crow Creek & & & & 0 & 0 \\
\hline 70 & Crow Creek & 44 & 0 & 0 & 0 & 0 \\
\hline 71 & Sage Creek & & & & 0 & 0 \\
\hline 72 & Deer Creek & & & & 0 & 1 \\
\hline 73 & White Dugway Creek & & & & 0 & 0 \\
\hline \multicolumn{7}{|c|}{ Teton River drainage } \\
\hline 74 & Teton River & & & & 80 & 46 \\
\hline 75 & Teton River & & & & 64 & 34 \\
\hline 76 & Teton River & & & & 41 & 28 \\
\hline 77 & Teton River & 63 & 20.8 & 17.7 & 11 & 16 \\
\hline
\end{tabular}

${ }^{\mathrm{a}} \mathrm{NF}=$ North Fork, $\mathrm{MF}=$ Middle Fork, and $\mathrm{SF}=$ South Fork.

b Based on fish $<10 \mathrm{~cm}$ at site.

$(82 \%)$ as in $1999-2000$ (78\%; Table 1). Yellowstone cutthroat trout made up $100 \%$ of the catch at 33 sites in the 1980 s, compared with $100 \%$ at 25 sites in 1999-2000. The proportion of rainbow trout and hybrids in the catch also did not change, averaging $7 \%$ in the 1980 s and in 1999-2000, but the number of locations where rainbow trout and hybrids were present increased from 23 to 37 sites (Table 1). Most (76\%) of the expanded distribution of rainbow trout and hybrids occurred in the Blackfoot River drainage (41\% of the expansion) and in two tributaries (Burns Creek and Pine Creek, including the North Fork) of the South Fork Snake River (35\%). Rainbow trout and hybrids made up less than $10 \%$ of the catch at 67 sites in the 1980 s and at 59 sites in 1999-2000. Brown trout and brook trout were present in 20 and 18 sites in the 1980s and in 15 and 8 sites in 1999-2000, respectively.

In general, genetic results corroborated our visual assessment of the rates of introgression at our study sites. At the 12 sites where no hybridization was visually identified and genetic results were available, no hybridization was detected in the nDNA or mtDNA of fish from 11 of the sites, whereas a rainbow trout allele was detected in the nDNA of one fish at the other site (Table 3). Of the five sites that did contain rainbow trout and hybrids based on visual identification and for which genetic results were available, percent introgression averaged $12 \%$ for visual identification, $16 \%$ for nDNA, and $11 \%$ for mtDNA (Table 3).

Yellowstone cutthroat trout size structure also remained relatively unchanged from the 1980s to 1999-2000 (Table 4). At the 48 sites where Yellowstone cutthroat trout sample sizes were large enough to calculate size structure for both periods, there was a slight but significant decrease from the 1980 s to $1999-2000$ in the proportion of fish that were $10-20 \mathrm{~cm}$ long $(74 \%$ versus $66 \% ; \bar{d}=-8 \pm$ $7 ; t=-3.13 ; P=0.003)$, but the proportion of fish $20-30 \mathrm{~cm}(16 \%$ versus $18 \% ; \bar{d}=2 \pm 6 ; t=$ $0.74 ; P=0.47), 30-40 \mathrm{~cm}(8 \%$ versus $12 \% ; \bar{d}=$ $4 \pm 5 ; t=2.21 ; P=0.03)$, and longer than $40 \mathrm{~cm}$ ( $3 \%$ versus $4 \% ; \bar{d}=2 \pm 3 ; t=1.60 ; P=0.12$ ) were not different between time periods. The slight 
TABLE 4.-Comparison of Yellowstone cutthroat trout size structure at study sites across southeastern Idaho between the 1980s and 1999-2000. Some of the 77 sites sampled did not contain enough Yellowstone cutthroat trout to report any size structure data for a particular time period.

\begin{tabular}{|c|c|c|c|c|c|c|c|c|c|}
\hline \multirow[b]{3}{*}{ Site } & \multirow[b]{3}{*}{ Stream ${ }^{a}$} & \multicolumn{8}{|c|}{ Percent of Yellowstone cutthroat trout catch per size category $(\mathrm{cm})$} \\
\hline & & \multicolumn{4}{|c|}{$1980 \mathrm{~s}$} & \multicolumn{4}{|c|}{$1999-2000$} \\
\hline & & $10-20$ & $20-30$ & $30-40$ & $>40$ & $10-20$ & $20-30$ & $30-40$ & $>40$ \\
\hline \multicolumn{10}{|c|}{ Portneuf River drainage } \\
\hline 5 & Pebble Creek & 92 & 8 & 0 & 0 & 97 & 3 & 0 & 0 \\
\hline 6 & Pebble Creek & 90 & 10 & 0 & 0 & 68 & 30 & 2 & 0 \\
\hline 7 & Pebble Creek & 97 & 3 & 0 & 0 & 74 & 26 & 0 & 0 \\
\hline 8 & Pebble Creek, NF & 100 & 0 & 0 & 0 & 95 & 5 & 0 & 0 \\
\hline 14 & Toponce Creek, SF & 74 & 26 & 0 & 0 & 83 & 17 & 0 & 0 \\
\hline 15 & Toponce Creek, SF & 91 & 9 & 0 & 0 & 93 & 7 & 0 & 0 \\
\hline \multicolumn{10}{|c|}{ Blackfoot River drainage } \\
\hline 16 & Blackfoot River & 14 & 50 & 14 & 21 & 15 & 53 & 5 & 27 \\
\hline 17 & Blackfoot River & 91 & 6 & 3 & 0 & 95 & 2 & 1 & 2 \\
\hline 18 & Blackfoot River & 35 & 27 & 25 & 13 & 43 & 8 & 18 & 31 \\
\hline 20 & Diamond Creek & 94 & 6 & 0 & 0 & 92 & 8 & 0 & 0 \\
\hline 21 & Diamond Creek & 98 & 2 & 0 & 0 & 97 & 3 & 0 & 0 \\
\hline 22 & Diamond Creek & 97 & 3 & 0 & 0 & 99 & 1 & 0 & 0 \\
\hline 23 & Diamond Creek & & & & & 100 & 0 & 0 & 0 \\
\hline 24 & Diamond Creek & 97 & 3 & 0 & 0 & 96 & 0 & 4 & 0 \\
\hline 25 & Diamond Creek & 93 & 7 & 0 & 0 & 98 & 2 & 0 & 0 \\
\hline \multicolumn{10}{|c|}{ Willow Creek drainage } \\
\hline 27 & Willow Creek & 22 & 67 & 7 & 3 & 14 & 36 & 45 & 5 \\
\hline 28 & Willow Creek & 76 & 23 & 1 & 0 & 51 & 45 & 4 & 0 \\
\hline 29 & Brockman Creek & & & & & 4 & 30 & 65 & 0 \\
\hline 30 & Corral Creek & 100 & 0 & 0 & 0 & & & & \\
\hline 31 & Corral Creek & 97 & 3 & 0 & 0 & 100 & 0 & 0 & 0 \\
\hline \multicolumn{10}{|c|}{ Main-stem Snake River drainage } \\
\hline 32 & Snake River & 1 & 31 & 54 & 14 & 4 & 45 & 36 & 15 \\
\hline \multicolumn{10}{|c|}{ South Fork Snake River drainage } \\
\hline 33 & Snake River, SF & 3 & 24 & 51 & 23 & 5 & 22 & 63 & 11 \\
\hline 34 & Snake River, SF & 2 & 19 & 59 & 20 & 6 & 32 & 55 & 7 \\
\hline 35 & Snake River, SF & 2 & 8 & 78 & 13 & 4 & 34 & 59 & 3 \\
\hline 36 & Burns Creek & 89 & 11 & 0 & 0 & 96 & 0 & 4 & 0 \\
\hline 37 & Burns Creek & & & & & 63 & 30 & 7 & 0 \\
\hline 38 & Pine Creek & 91 & 9 & 0 & 0 & 98 & 2 & 0 & 0 \\
\hline 39 & Pine Creek & 84 & 13 & 3 & 0 & 100 & 0 & 0 & 0 \\
\hline 40 & Pine Creek & 97 & 3 & 0 & 0 & 87 & 12 & 1 & 0 \\
\hline 41 & Pine Creek & 83 & 14 & 2 & 0 & 94 & 6 & 0 & 0 \\
\hline 43 & Pine Creek, NF & 91 & 9 & 0 & 0 & & & & \\
\hline 44 & Rainey Creek & & & & & 73 & 25 & 2 & 0 \\
\hline 46 & Rainey Creek & & & & & 41 & 38 & 20 & 1 \\
\hline 47 & Fall Creek & & & & & 60 & 37 & 3 & 0 \\
\hline 48 & Bear Creek & 100 & 0 & 0 & 0 & 94 & 5 & 0 & 1 \\
\hline 49 & Elk Creek & 96 & 4 & 0 & 0 & 92 & 8 & 0 & 0 \\
\hline 50 & Big Elk Creek & & & & & 16 & 9 & 53 & 22 \\
\hline 51 & Big Elk Creek & 12 & 44 & 36 & 8 & 4 & 11 & 62 & 24 \\
\hline 52 & McCoy Creek & 93 & 6 & 0 & 1 & 80 & 16 & 4 & 1 \\
\hline 53 & McCoy Creek & 95 & 5 & 0 & 0 & 93 & 5 & 2 & 0 \\
\hline 54 & McCoy Creek & 94 & 6 & 0 & 0 & 91 & 8 & 1 & 0 \\
\hline 55 & Jensen Creek & 88 & 12 & 0 & 0 & & & & \\
\hline 56 & Fish Creek & 100 & 0 & 0 & 0 & 46 & 53 & 1 & 0 \\
\hline 57 & Fish Creek & 94 & 3 & 3 & 0 & & & & \\
\hline 58 & Barnes Creek & 87 & 13 & 0 & 0 & 93 & 7 & 0 & 0 \\
\hline 60 & Clear Creek & 93 & 7 & 0 & 0 & 95 & 5 & 0 & 0 \\
\hline 61 & Iowa Creek & 96 & 4 & 0 & 0 & 100 & 0 & 0 & 0 \\
\hline 62 & Jackknife Creek & 81 & 16 & 3 & 0 & & & & \\
\hline 63 & Tincup Creek & 88 & 12 & 0 & 0 & 42 & 47 & 11 & 0 \\
\hline 64 & Tincup Creek & 93 & 7 & 0 & 0 & 80 & 17 & 3 & 0 \\
\hline 65 & Tincup Creek & 97 & 3 & 0 & 0 & 81 & 14 & 5 & 0 \\
\hline
\end{tabular}


TABLE 4.-Continued.

\begin{tabular}{|c|c|c|c|c|c|c|c|c|c|}
\hline \multirow[b]{3}{*}{ Site } & \multirow[b]{3}{*}{ Stream ${ }^{\mathrm{a}}$} & \multicolumn{8}{|c|}{ Percent of Yellowstone cutthroat trout catch per size category $(\mathrm{cm})$} \\
\hline & & \multicolumn{4}{|c|}{$1980 \mathrm{~s}$} & \multicolumn{4}{|c|}{$1999-2000$} \\
\hline & & $10-20$ & $20-30$ & $30-40$ & $>40$ & $10-20$ & $20-30$ & $30-40$ & $>40$ \\
\hline 66 & Bear Canyon Creek & 100 & 0 & 0 & 0 & & & & \\
\hline 67 & Stump Creek & 86 & 13 & 1 & 0 & 67 & 30 & 2 & 0 \\
\hline 68 & Horse Creek & 86 & 14 & 0 & 0 & 90 & 10 & 0 & 0 \\
\hline 69 & Crow Creek & & & & & 24 & 41 & 34 & 0 \\
\hline 70 & Crow Creek & 78 & 22 & 0 & 0 & 83 & 17 & 0 & 0 \\
\hline 71 & Sage Creek & 72 & 28 & 0 & 0 & 34 & 64 & 2 & 0 \\
\hline 72 & Deer Creek & 80 & 18 & 2 & 0 & 80 & 19 & 1 & 0 \\
\hline \multicolumn{10}{|c|}{ Teton River drainage } \\
\hline 74 & Teton River & 45 & 43 & 8 & 5 & 2 & 40 & 39 & 19 \\
\hline 75 & Teton River & 42 & 51 & 5 & 2 & 4 & 15 & 56 & 26 \\
\hline 76 & Teton River & 50 & 43 & 6 & 1 & 9 & 20 & 50 & 22 \\
\hline 77 & Teton River & 53 & 42 & 4 & 1 & 16 & 41 & 32 & 11 \\
\hline
\end{tabular}

${ }^{\mathrm{a}} \mathrm{NF}=$ North Fork, SF $=$ South Fork.

decrease in the proportion of fish $10-20 \mathrm{~cm}$ long was caused by the large shift observed at the Teton River sites, where the percentage of fish $10-20 \mathrm{~cm}$ long decreased from an average of $48 \%$ to $8 \%$ between the 1980s and 1999-2000, and fish larger than $30 \mathrm{~cm}$ increased from an average of $8 \%$ to $64 \%$. Excluding these data, the difference between the proportion of fish $10-20 \mathrm{~cm}$ in the $1980 \mathrm{~s}(76 \%)$ and $1999-2000(72 \%)$ was not statistically significant $(\bar{d}=-5 \pm 6 ; t=-2.17 ; P=0.04)$.

\section{Discussion}

Yellowstone cutthroat trout abundance and distribution in Idaho has undoubtedly declined over the last century. For example, we know of only 12 streams in the Henry's Fork Snake River drainage (excluding the Teton River drainage) that currently contain Yellowstone cutthroat trout. Similarly, Yellowstone cutthroat trout are scarce across much of the Raft River, Goose Creek, Bannock Creek, and Rock Creek drainages, although assessing the historical distribution or abundance of Yellowstone cutthroat trout in these drainages is problematic because they currently contain, and probably historically contained, few perennial streams. In the Snake River from Shoshone Falls to Idaho Falls, Yellowstone cutthroat trout are either absent or persisting at low densities. In general, these declines appear to have been the result of displacement by nonnative salmonids (rainbow trout, brook trout, and brown trout) or other nonnative game fish or of major habitat alterations, whether in the main stem of the Snake River or in headwater tributaries. Because our sample sites in the 1980s were selected not at random but as part of IDFG's general inventory sampling, some caution in extrapolating the results of this study throughout southeastern Idaho is warranted. However, the broad geographic nature of our monitoring effort, and the wide array of habitats sampled (from small creeks to large rivers), suggests that in general Yellowstone cutthroat trout abundance over the last 10-20 years in Idaho has remained relatively stable.

The distribution of Yellowstone cutthroat trout in our study sites also remained relatively stable from the 1980s to the present. We failed to capture Yellowstone cutthroat trout longer than $10 \mathrm{~cm}$ at only five sites that previously contained them. In 1986, the site on Jensen Creek included a large beaver pond that had breached by 1999 , leaving a shallow, uncomplex section of stream that nonetheless contained several Yellowstone cutthroat trout smaller than $10 \mathrm{~cm}$; moreover, Yellowstone cutthroat trout longer than $10 \mathrm{~cm}$ were present upstream and downstream of the site. The lower site on Toponce Creek was a degraded section of stream (180 m long) that contained one Yellowstone cutthroat trout in 1986 and none in 2001. In Birch Creek and Cold Creek, Yellowstone cutthroat trout were sparse in 1987 ; by 2000 , the species appeared to have been completely replaced by brook trout. Our study design precluded definitive conclusions regarding changes in Yellowstone cutthroat trout distribution outside the study sites sampled. Indeed, because all sites in our study contained Yellowstone cutthroat trout in the 1980s, we could have detected only range contractions, not expansions.

Despite the implementation of restrictive harvest regulations for Yellowstone cutthroat trout in Idaho, we found no increase in size of Yellowstone cutthroat trout between the two sampling periods ex- 
cept at the Teton River sites. Regulations restricting harvest of Yellowstone cutthroat trout were initially implemented in the South Fork Snake River in 1984. By 1990, some form of angler harvest restrictions were in place for Yellowstone cutthroat trout in all major drainages in Idaho. Restrictions have focused on protecting spawning-size fish and have included size limits, bag limits, slot limits, and delayed openings of the fishing season in spawning streams. Except at the Teton River sites, these changes have not led to an increase in fish size, at least when comparing the sites in our study. Previous studies of size and slot limit strategies have not shown consistent improvements in stock structure (see review in Power and Power 1996).

Because salmonid populations often experience dramatic fluctuations in abundance, both temporally (Platts and Nelson 1988; House 1995) and spatially (Milner et al. 1993), detecting changes or trends in salmonid populations can be difficult (e.g., Rieman and Myers 1997). Such population fluctuations are often the result of environmental fluctuations, such as floods, low flows, or severe winter conditions, which temporally vary in frequency as well. Because our sampling during both periods occurred over multiple years, the potential spatial and temporal fluctuations in fish abundances that make before-after analyses difficult may have been somewhat mitigated by dispersing such environmental fluctuations over more than 1 year of sampling. Platts and Nelson (1988) and Bohlin et al. (1989) suggested that a study design such as ours, with paired comparisons incorporating more than 1 year of sampling, would be more likely to detect changes in trout populations if they occurred. We recommend that these sites be monitored regularly in the future to further elucidate trends in Yellowstone cutthroat trout abundance, distribution, and size structure.

Rainbow trout and hybrids were present in almost half of the comparison sites in 1999-2000. However, we urge caution in extrapolating hybridization results to other areas in Idaho containing Yellowstone cutthroat trout for two reasons. First, most of the expansion was concentrated in the Blackfoot River drainage and in two tributaries of the South Fork Snake River. Outside of these 17 sites, rainbow trout and hybrids were present at 21 of the remaining 60 sites in the 1980 s, compared with 22 sites in 1999-2000, making up an average of $9 \%$ of the catch in the 1980s and 6\% in 1999-2000. Second, recent large-scale random sampling in southeast Idaho suggests that Yellowstone cutthroat trout hybridization may be less widespread than this study would indicate. For example, in this study, rainbow trout and hybrids were captured in $73 \%$ of the sites in the Portneuf River drainage and all sites in the Teton River in 1999-2000. But in 2000-2001, when IDFG personnel also sampled 88 randomly distributed sites in the Portneuf River drainage and 90 randomly distributed sites in the Teton River drainage, they found rainbow trout and hybrids in only $17 \%$ and $8 \%$, respectively, of the sites that contained Yellowstone cutthroat trout (K. A. Meyer, unpublished data). Such discrepancies may be a reflection of the sampling design used in our present comparison. In general, sites for this study were established in the 1980s in the lower segments of streams with greater angling pressure, where rainbow trout were more often stocked and where subsequent hybridization was more likely to develop. Nevertheless, we agree with the assertion by Kruse et al. (2000) that controlling hybridization is an important factor in assuring the long-term persistence of Yellowstone cutthroat trout. Management activities, such as stocking only sterile triploid rainbow trout in the few places where hatchery stocking within the historical range of Yellowstone cutthroat trout still occurs (Dillon et al. 2000), and large-scale removals of rainbow trout and hybrids through electrofishing, trapping of migrating spawners, and liberalized regulations for rainbow trout and hybrids have been implemented and are being evaluated throughout southeastern Idaho.

Our study design had important limitations. The lack of randomization in site selection makes any extrapolation of Yellowstone cutthroat trout abundance and size structure beyond the areas that we sampled problematic. In addition, the visual identification of hybridization we used at most sites was less reliable than genetic analysis would have been, although the genetic results we report do support our phenotypic identifications, and recent studies have demonstrated the accuracy that can be achieved in identifying Yellowstone cutthroat trout $\times$ rainbow trout hybrids with phenotypic characteristics (Kruse 1998; Campbell et al. 2002). Lastly, using block nets at many of the removal sites in 1999-2000 that in the 1980s were not sampled with block nets may have resulted in biased estimates between time periods at these sites. However, we do not believe that the block net differences affected our results appreciably for several reasons. First, site boundaries were placed in riffles where swift streamflow probably discouraged upstream escapement by fish. Second, we saw no indication of a concentration of salmonids in 
the vicinity of the block nets at the sites where nets were used. Third, the discrepancy involved only $51 \%$ of the sites, and we found no pattern of increased or decreased abundance related to whether or not block nets had been used in 19992000. Finally, as others have previously suggested, the use of block nets in small streams may not be necessary for territorial salmonids (Bohlin et al. 1989; Simonson and Lyons 1995).

Despite these limitations, our results indicate that Yellowstone cutthroat trout abundance, distribution, and stock structure have remained relatively unchanged from the 1980s to 1999-2000 at a large number of locations across their historical range in Idaho. However, during the study periods, we found that rainbow trout and hybrid distribution expanded in two major drainages. Continued monitoring of trout populations, in the reaches we monitored and in a more widely distributed sample, will be necessary to determine rangewide trends in Yellowstone cutthroat trout populations in Idaho.

\section{Acknowledgments}

We thank Tim Burton, Chip Corsi, Jeff Dillon, Scott Grunder, Tony Lamansky, Liz Mamer, Virgil Moore, and Russ Thurow for helping to relocate the 1980s sites and for data collection and compilation. Robert Gresswell, Brad Shepard, Dave Zafft, and an anonymous reviewer provided excellent comments and suggestions that greatly improved the manuscript. This project was supported by funds from Federal Aid in Sport Fish Restoration, project $\mathrm{F}-73-\mathrm{R}-20$, and by the Bonneville Power Administration.

\section{References}

Behnke, R. J. 1992. Native trout of western North America. American Fisheries Society, Monograph 6, Bethesda, Maryland.

Bohlin, T., S. Hamrin, T. G. Heggberget, G. Rasmussen, and S. J. Saltveit. 1989. Electrofishing-theory and practice with special emphasis on salmonids. Hydrobiologia 173:9-43.

Campbell, M. R., J. Dillon, and M. S. Powell. 2002. Hybridization and introgression in a managed, native Yellowstone cutthroat trout Oncorhynchus clarki bouvieri population, Henrys Lake, Idaho: genetic detection and management implications. Transactions of the American Fisheries Society 131:364375.

Decker, L. M., and D. C. Erman. 1992. Short-term seasonal changes in composition and abundance of fish in Sagehen Creek, California. Transactions of the American Fisheries Society 121:297-306.

Dillon, J., D. Schill, D. Teuscher, and D. Megargle. 2000.
Triploid hatchery trout programs in Idaho-meeting public demand for consumptive angling while protecting genetic integrity of native trout. Pages 105 108 in D. Schill, S. Moore, P. Byorth, and Bob Hamre, editors. Wild trout VII. Yellowstone National Park, Wyoming.

Gresswell, R. E. 1995. Yellowstone cutthroat trout. Pages 36-54 in M. K. Young, editor. Conservation assessment for inland cutthroat trout. U.S. Forest Service General Technical Report RM-GTR-256.

House, R. 1995. Temporal variation in abundance of an isolated population of cutthroat trout in western Oregon, 1981-1991. North American Journal of Fisheries Management 15:33-41.

Johnson, D. H. 1995. Statistical sirens: the allure of nonparametrics. Ecology 76:1998-2000.

Kruse, C. G. 1998. Influence of non-native trout and geomorphology on distributions of indigenous trout in the Yellowstone River drainage of Wyoming. Doctoral dissertation. University of Wyoming, Laramie.

Kruse, C. G., W. A. Hubert, and F. J. Rahel. 2000. Status of Yellowstone cutthroat trout in Wyoming waters. North American Journal of Fisheries Management 20:693-705.

May, B. E. 1996. Yellowstone cutthroat trout, Oncorhynchus clarki bouvieri. Pages 11-34 in D. A. Duff, editor. Conservation assessment for inland cutthroat trout: distribution, status and habitat management implications. U.S. Forest Service, Ogden, Utah.

Milner, N. J., R. J. Wyatt, and M. D. Scott. 1993. Variability in the distribution and abundance of stream salmonids, and the associated use of habitat models. Journal of Fish Biology 43(Supplement A):103119.

Montana Fish, Wildlife and Parks. 1997. Mark recapture for Windows, version 5.0. Montana Fish, Wildlife and Parks, Bozeman.

Platts, W. S., and R. L. Nelson. 1988. Fluctuations in trout populations and their implications for landuse evaluation. North American Journal of Fisheries Management 8:333-345.

Power, M., and G. Power. 1996. Comparing minimumsize and slot limits for brook trout management. North American Journal of Fisheries Management 16:49-62.

Rieman, B. E., and D. L. Myers. 1997. Use of redd counts to detect trends in bull trout (Salvelinus confluentus) populations. Conservation Biology 11: 1015-1018.

Simonson, T. D., and J. Lyons. 1995. Comparison of catch per effort and removal procedures for sampling stream fish assemblages. North American Journal of Fisheries Management 15:419-427.

Simpson, J. C., and R. L. Wallace. 1982. Fishes of Idaho. University of Idaho Press, Moscow.

Sokal, R. R., and F. J. Rohlf. 1987. Introduction to biostatistics, 2nd edition. Freeman, New York.

Thurow, R. F., C. E. Corsi, and V. K. Moore. 1988. Status, ecology, and management of Yellowstone cutthroat trout in the upper Snake River drainage, Idaho. Pages 25-36 in R. E. Gresswell, editor. Status 
and management of interior stocks of cutthroat trout. American Fisheries Society, Symposium 4, Bethesda, Maryland.

Thurow, R. F., D. C. Lee, and B. E. Rieman. 1997. Distribution and status of seven native salmonids in the interior Columbia River Basin and portions of the Klamath River and Great basins. North American Journal of Fisheries Management 17:1094-1110.

USFWS (U.S. Fish and Wildlife Service). 2001. 90-day finding for a petition to list the Yellowstone cutthroat trout as threatened. Federal Register 66: (23 February 2001) 11244-11249.
Van Deventer, J., and W. S. Platts. 1989. Microcomputer software system for generating population statistics from electrofishing data-user's guide for MicroFish 3.0. U.S. Forest Service General Technical Report INT-254.

Varley, J. D., and R. E. Gresswell. 1988. Ecology, status, and management of the Yellowstone cutthroat trout. Pages 13-24 in R. E. Gresswell, editor. Status and management of interior stocks of cutthroat trout. American Fisheries Society, Symposium 4, Bethesda, Maryland.

Zar, J. H. 1996. Biostatistical analysis, 3rd edition. Prentice-Hall, Upper Saddle River, New Jersey. 